\title{
3D Scanning of Dutch Military Secular Trends in PCA for 18,000 Soldiers
}

\author{
Frank B. TER HAAR ${ }^{a}$, Henk G.B. REULINK ${ }^{b}$, Hein A.M. DAANEN ${ }^{\star a, c, d, e}$ \\ ${ }^{a}$ TNO, Soesterberg and The Hague, The Netherlands; \\ ${ }^{\mathrm{b}}$ Clothing and Personal Equipment Office, Ministry of Defence, Soesterberg, The Netherlands; \\ 'MOVE Research Institute, Faculty of Human Movement Sciences, VU University, Amsterdam; \\ ${ }^{\mathrm{d}}$ Sizing Science, Soesterberg, The Netherlands; \\ ${ }^{\mathrm{e}} \mathrm{AMFI}$ - Amsterdam Fashion Institute, CREATE-IT applied research, Amsterdam University of Applied \\ Sciences, Amsterdam, The Netherlands \\ http://dx.doi.org/10.15221/13.144
}

\begin{abstract}
The Netherlands Military Forces started 3D whole body scanning of their recruits in the year 2003. 1D-body dimensions are derived from the scans to supply the best fitting size without human involvement. In order to investigate secular trends in body dimensions, the dataset of 3D scans was cleaned and analyzed for inter-individual differences using principle component (PC) analysis. Changes in body dimensions and body posture were quantified over the period 2003-2012 and digital design extremes were constructed.

The original dataset of 18221 scans was cleaned to 15231 male and 2390 female scans (97\%). A male and female model was constructed from 200 selected men and 200 selected women. Since the arm position was not standardized during scanning, it was necessary to separate the arms from the torso. The analysis showed that men and women differed most for stature (PC1), mass and inner leg length (PC2) and mass without inner leg length (PC3). These PC values hardly changed over the years. However, the PCs of the arms changed over the years due to changing preferences in posture.

The developed model enables quantification of the body shape and posture with only a small number of PCs. This means that the data can be stored and processed effectively. The generated design extremes may serve to optimize products related to the physical shape of soldiers.
\end{abstract}

Keywords: Principle Component Analysis, full body scans, clothing

\section{Introduction}

The armed forces have the obligation to supply fitting combat apparel to every soldier. Misfits result in suboptimal performance, movement limitations, thermal problems due to insufficient ventilation and so on. Fitting the apparel is a time consuming and thus expensive process. The Netherlands armed forces tried to reduce the costs by introducing 3D whole body scanning in 2003 . Whole body scanners make a copy of the outside of the body, from which secondary measures like circumferences, volume and surface area can be derived [1]. One of the first application areas of whole body scanning was the military domain [2], and The Netherlands was one of the first countries to start with this technique.

3D scans offer a wide potential of applications, far beyond the simple deduction of 1D measures from the 3D shape [3]. The Netherlands armed forces are interested in this potential and in particular the analysis of 3D shapes. The analysis of 3D shapes has increased considerably since the 3D scans of the CAESAR survey [4]. In particular the Principal Component Analysis (PCA) contributed to improved processing of the 3D scans, repairing occluded areas and quantifying shape $[5,6]$.

The particular interest in processing the scans is 1) to generate a clean database of 3D-scans, 2) to identify changes over time in the PC of the 3D scans and 3) to generate design extremes for design and evaluation of new products that have to fit the Dutch soldier.

In this article, the methods and main findings are described.

* hein.daanen@tno.nl; +31-888665948; www.tno.nl ; www.sizingscience.nl 


\section{Methods}

\subsection{Scanner and subjects}

The subjects were military recruits from the Netherlands Armed Forces. They were all scanned in standing position in the Vitronic Smart system (www.vitronic.de). 87 Body dimensions were derived from the 3D scans using Human Solutions software. Each scan had a date stamp and was visually checked for gender and error. The scan was coded by the military number of the subject that included the day of birth.

\subsection{Database}

The scanner generates a folder for every scanned subject. 18610 Folders were available from week 21 in 2003 to week 46 in 2012. 18319 folders contained a scan-file and a file with derived body dimensions.

Figure1 shows the number of scans for each year. Due to the unavailability of the scanner in 2009 , only a few scans were made.

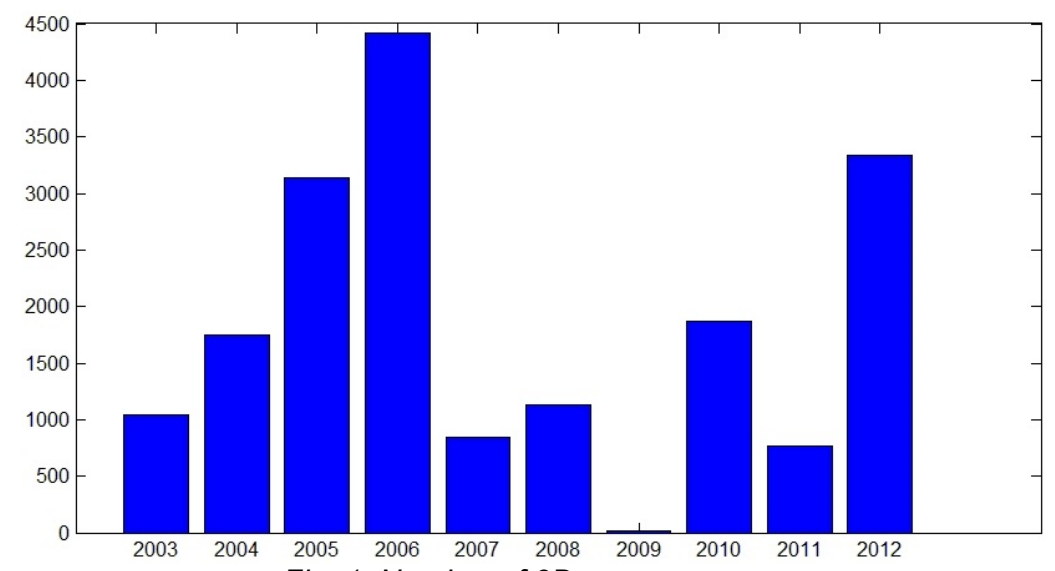

Fig. 1. Number of $3 D$ scans per year

The file size of each 3D scan is plotted in figure 2 . Several scans missed data, and they were removed from the database. After cleaning, 15231 male scans and 2390 female scan remained for analysis $(97 \%)$. Thus, $86 \%$ was male.
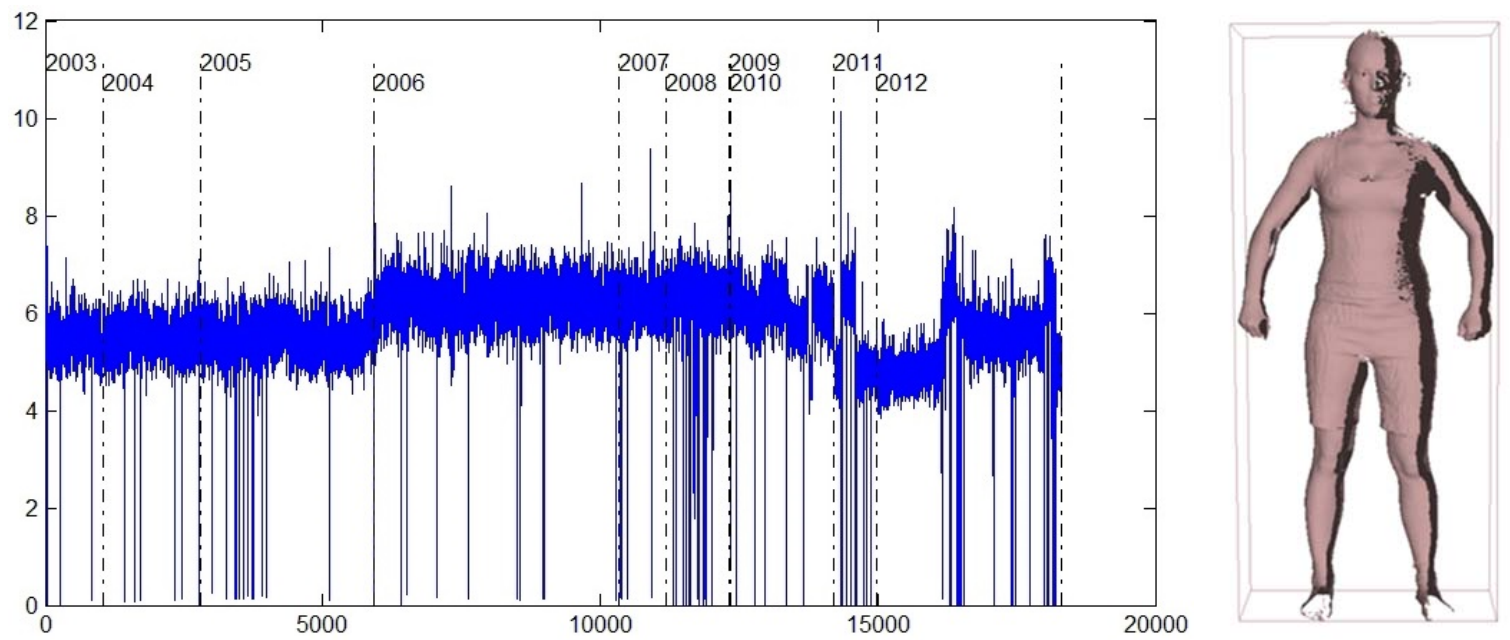

Fig. 2. File size of each scan (bytes $\times 10^{5}$ ). Please note that the file size after 2006 increased due to scanner improvements and that the size was reduced in 2011 and the first part of 2012 due to failure of a camera (see image at the right).

The next automatic cleaning procedure contained the cleaning based on incorrect filenames and birth dates. All scans with calculated ages above 65 years were removed.

Hereafter, a visual check was performed for each scan. Scans were removed with movement artifacts, black underwear (absorbing laser light), calibration objects, test scans etc. A final clean database remained with 17603 scans (95\% of the original number of folders). 


\subsection{Subject selection for model construction}

A male and female PCA model was created from a database of 200 selected male and 200 selected female scans. A grid of $5 \mathrm{~cm} \times 500 \mathrm{~cm}^{2}$ was superimposed over the stature / surface area plot (figure 3 ) and a subject from a grid cell was selected, resulting in 105 male and 63 female data points. The dataset was randomly increased to 200 subjects (figure 4 ).

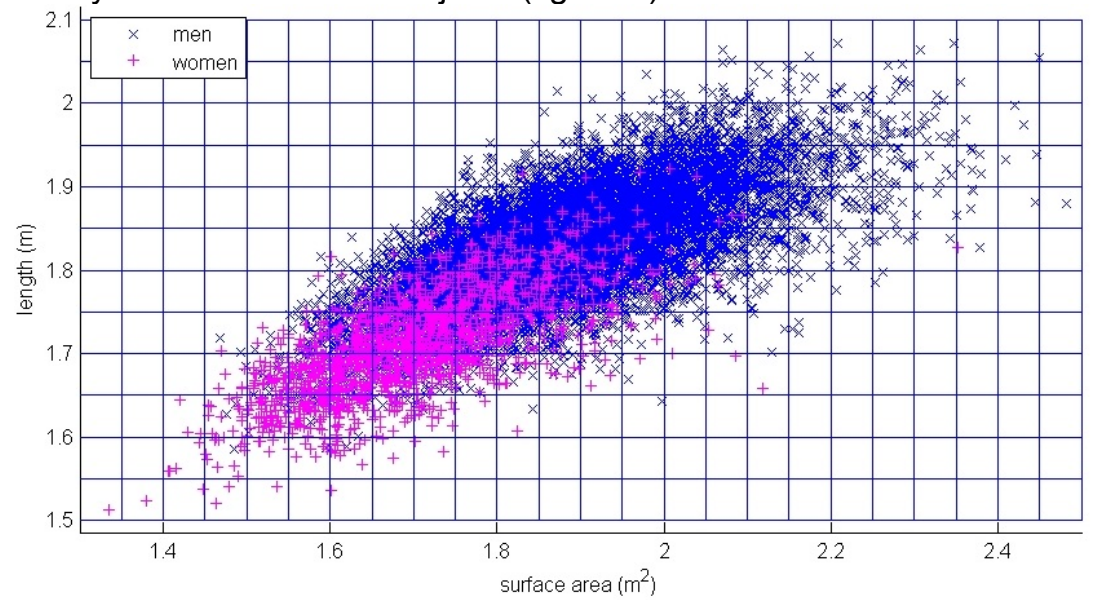

Fig. 3. Relation between stature (vertical axis) and body surface area (horizontal axis) for the male and female population.

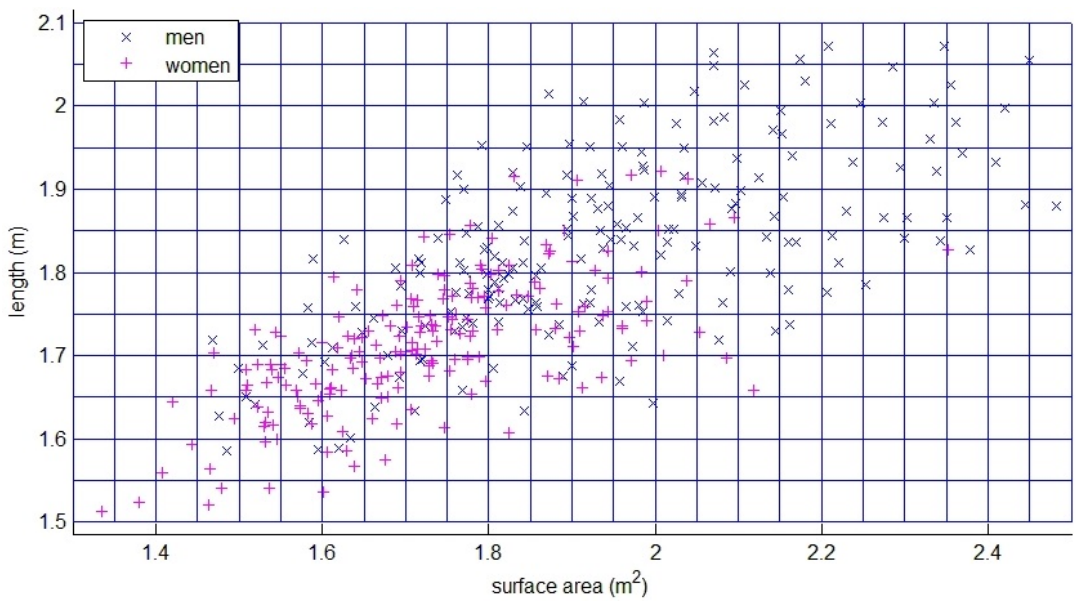

Fig. 4. Selected males and females for model construction.

\subsection{Model construction}

The 200 male and female scans were processed manually to generate the model. Corresponding points were identified and brought into full correspondence. Since the position of the arms was not standardized during the scan, it was a source of considerable variance, and it was decided to model the arms and body separately (Fig. 5).
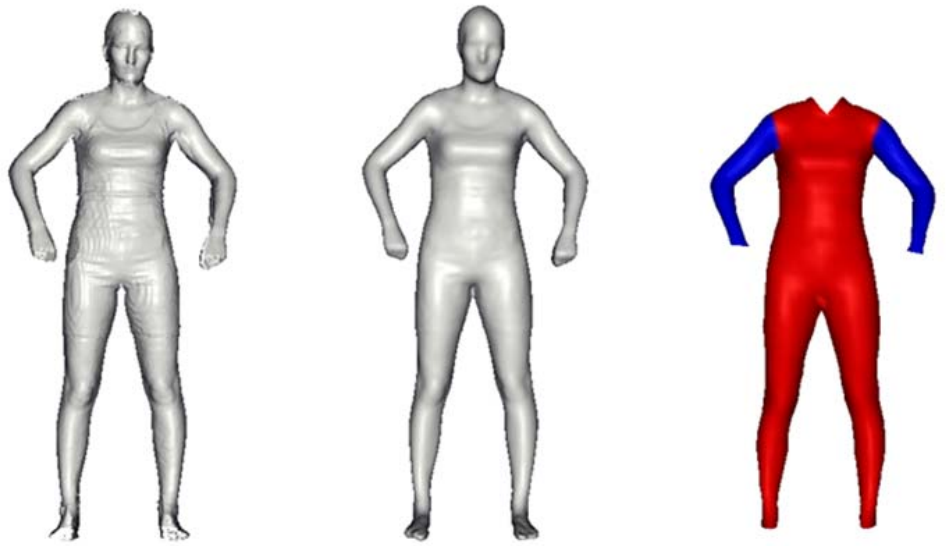

Fig. 5. Example of a raw 3D scan (left), a cleaned 3D scan (middle) and PCA model, in which body and arms were modeled separately. 


\subsection{Principal component analysis}

Principal component analysis (PCA) is a mathematical procedure searching for an orthogonal transformation of possibly correlated variables. After the PCA, the first PC represents maximal variation in the data, the second $P C$ the maximal variation orthogonal to the first $P C$, and so on until an orthogonal set of components is obtained for the $3 \mathrm{D}$ corresponding shape data. A PC is a list of $3 \mathrm{D}$ displacement vectors, that can be added or subtracted from the mean shape to visualize the (principal) change in the space of 3D body shapes [6].

For the analysis of the 3D dataset we need to have full correspondence for all male and female body scans. Therefore, we automatically fit the PCA models to each of the body scans with the same gender. The fitting process starts by placing the average model in a new 3D scan. Then the weights of the shape components are varied step by step and the resulting correspondence with the $3 \mathrm{D}$ scan is updated until dense correspondence is acquired. The list of eighty values for the first eighty PCs that provide the best fit is stored for the arms and body for each individual in the dataset, these eighty values characterize the body shape and the posture of the subject. These lists of PC values were added to the list of $1 \mathrm{D}$ body measures for trend analysis.

\section{Results}

\subsection{Fitting the 3D PCA models}

By definition the first PC explains most 3D shape variation and with each consecutive $P C$ the amount of explained variation gets less. Figure 6 shows the relation between PC-number and explained variance. It can be seen that after about 10 PCs, most of the 3D shape variance of the human body is explained, for men and women, body and arms.

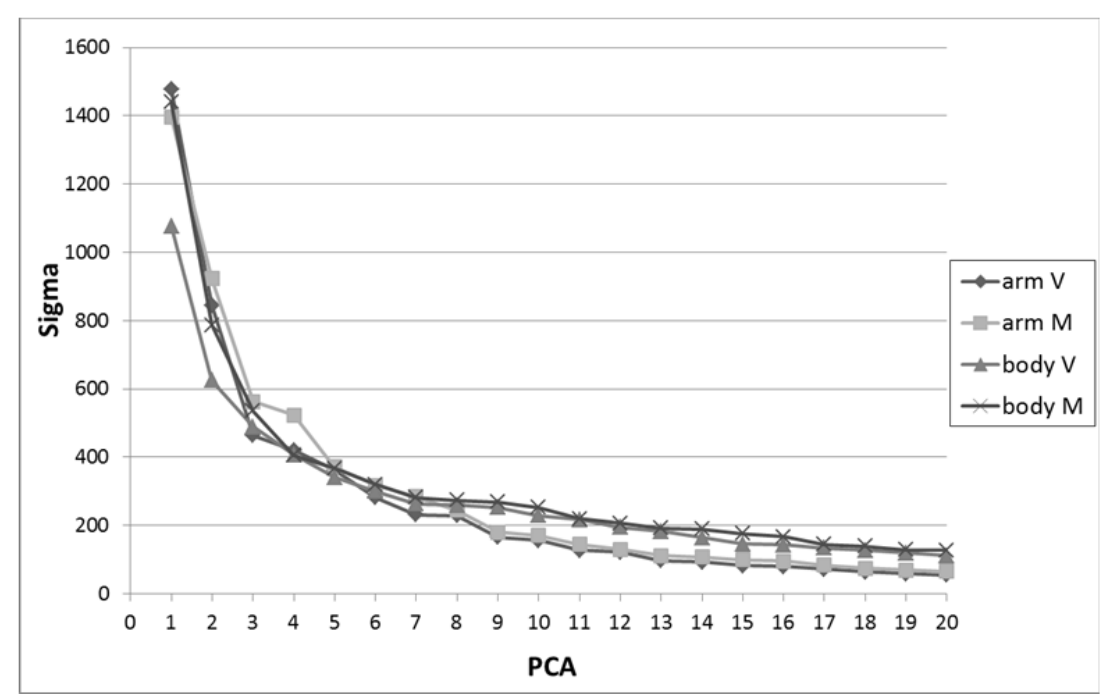

Fig. 6. The sigma values of the PCA model for the first 20 eigenvectors (PCs). The sigma represents the amount of $3 D$ change when the $3 D$ mean shape is adjusted along one of the PCs. Sigma is computed as the root of the

sum of the squared distances of the 3D points. If we consider a sphere with 2000 vertices and an PC1 that displaces each point of the mean sphere with $30 \mathrm{~mm}$, then the sigma equals 1341 for that PC.

The first principal component in both the male and female body is related to stature (figure 7).
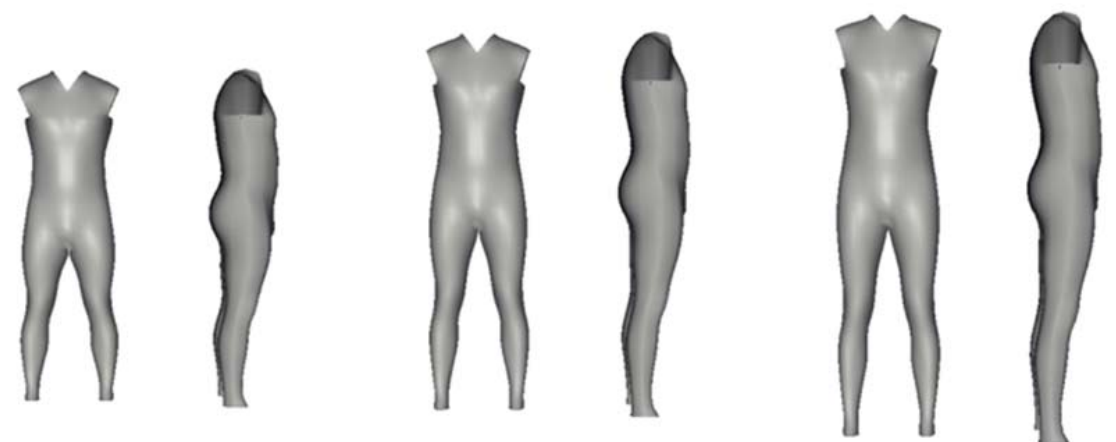

Fig. 7. Changes in the $1^{s t} P C$ for the male body model. Left: $-2^{*}$ Standard Deviation(SD), middle: 0 SD, right: $+2 S D$. 
For the arms, the first principal component is related to the abduction and anteflexion angle as show in figure 8.
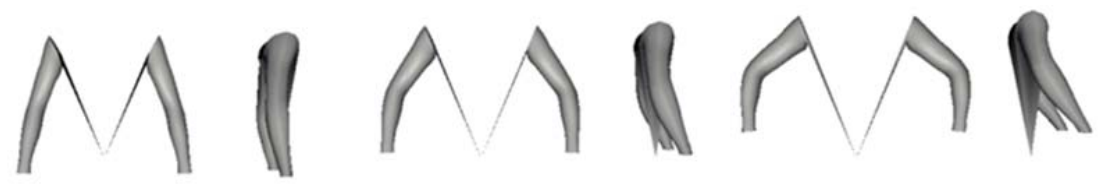

Fig. 8. Changes in the $1^{\text {st }} P C$ for the male arm model. Left: $-2 *$ Standard Deviation (SD), middle: $0 S D$, right: $+2 S D$.

Table 1 shows the relation between principal components and some main derived 1D body dimensions from the 3D scans. PC1 is clearly related to stature.

Table 1. Correlation between four scan derived body measures and the three principal components (PC) for male (M) and female (F) subjects, body and arm model.

\begin{tabular}{|l|l|l|c|c|c|c|}
\hline PC & body/arm & M/F & Stature & Hip circ. & Waist circ. & Inner leg length \\
\hline 1 & Body & M & 0.96 & 0.29 & 0.13 & 0.80 \\
\hline 2 & Body & M & -0.02 & 0.40 & 0.54 & -0.22 \\
\hline 3 & Body & M & 0.07 & 0.41 & 0.37 & 0.00 \\
\hline 1 & Body & F & 0.94 & 0.27 & 0.04 & 0.82 \\
\hline 2 & Body & F & -0.14 & 0.07 & 0.06 & -0.20 \\
\hline 3 & Body & F & 0.04 & 0.60 & 0.72 & 0.03 \\
\hline 1 & Arm & M & 0.28 & 0.28 & 0.22 & 0.21 \\
\hline 2 & Arm & M & 0.32 & 0.00 & -0.07 & 0.30 \\
\hline 3 & Arm & M & 0.13 & 0.22 & 0.30 & 0.11 \\
\hline 1 & Arm & F & 0.12 & 0.16 & 0.03 & 0.15 \\
\hline 2 & Arm & F & 0.26 & 0.11 & 0.03 & 0.26 \\
\hline 3 & Arm & F & 0.23 & 0.19 & 0.24 & 0.21 \\
\hline
\end{tabular}

\subsection{Trends in principal components}

How the principal components change over the years is investigated. The first principal component is related to stature. Stature itself hardly changed over the last decade (figure 9).

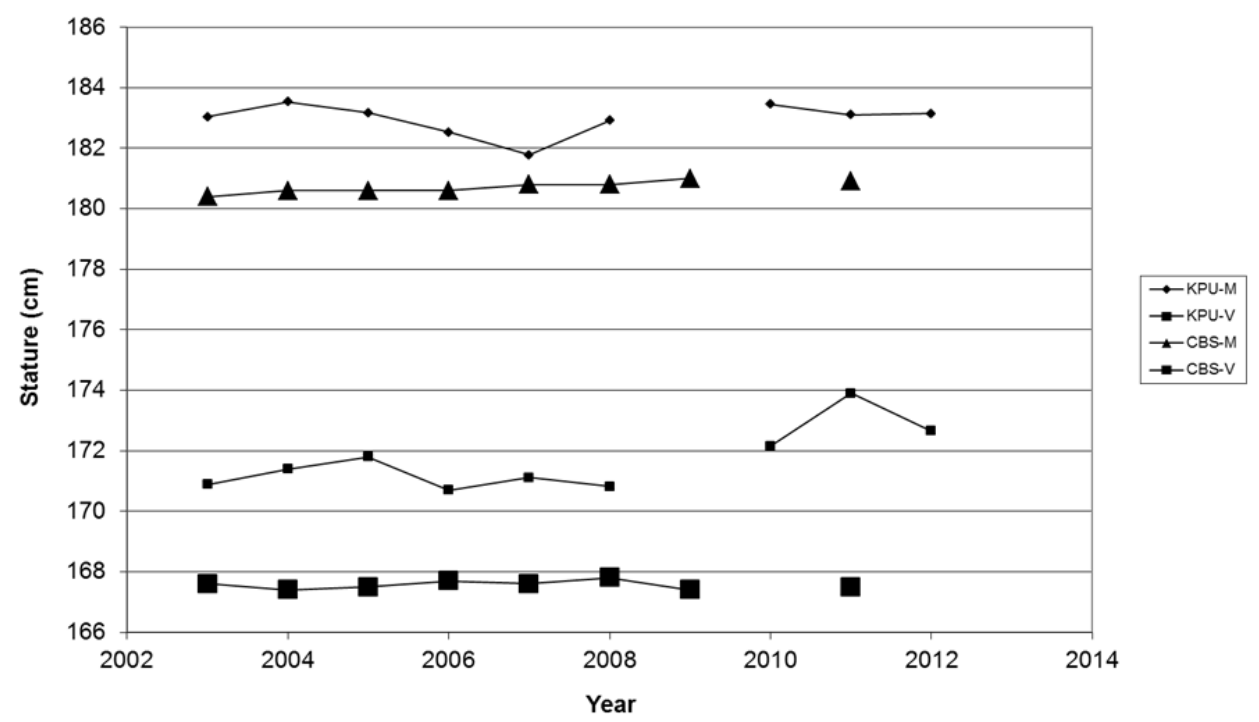

Fig. 9. Average stature (cm) for Dutch male (KPU-M) and female (KPU-V) recruits from 2003 to 2012. Data from 2009 were disregarded because the dataset was too small. As a comparison, the data from the National Statistics Office are added (CBS-M and CBS-V).

Correspondingly, the $1 \mathrm{st}$ principal component of the body was also rather stable (figure 10 ). The $2^{\text {nd }}$ PC of the body, related to body mass, was higher in the years 2003 and 2004. This was probably due to a relatively older population that was scanned during those years (age average above 30 years versus 20-28 years for the other years). The first and second principle components of the arms, however, changed over the years indicating that the position of the arms was not consistent. 


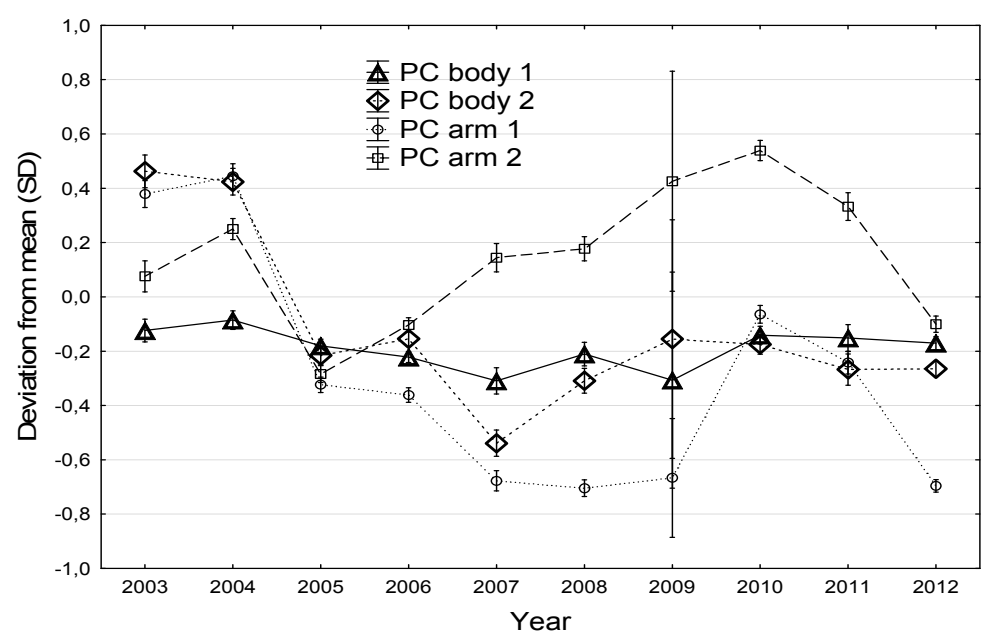

Fig. 10. Changes in the first two principal components of the arm and body over the years. The dataset of male

\subsection{Design extremes} and female subjects were combined in this graph.

For the design and evaluation of apparel, it is important to have a digital representation of design extremes. Nine design extremes were constructed, based on the first three principal components of the body, see Table 2 . We consider the mean shape to be a design extreme of the database as well.

Table 2. Design extremes with their weights for the PC components.

\begin{tabular}{|l|l|l|l|}
\hline & PC1 & PC2 & PC3 \\
\hline 1 & 0.00 & 0.00 & 0.00 \\
\hline 2 & -1.44 & -1.44 & -1.44 \\
\hline 3 & -1.44 & -1.44 & +1.44 \\
\hline 4 & -1.44 & +1.44 & -1.44 \\
\hline 5 & -1.44 & +1.44 & +1.44 \\
\hline 6 & +1.44 & -1.44 & -1.44 \\
\hline 7 & +1.44 & -1.44 & +1.44 \\
\hline 8 & +1.44 & +1.44 & -1.44 \\
\hline 9 & +1.44 & +1.44 & +1.44 \\
\hline
\end{tabular}

The arms were selected as a best match to the PC of the body. The nine design extremes for men are visualized in figure 11.
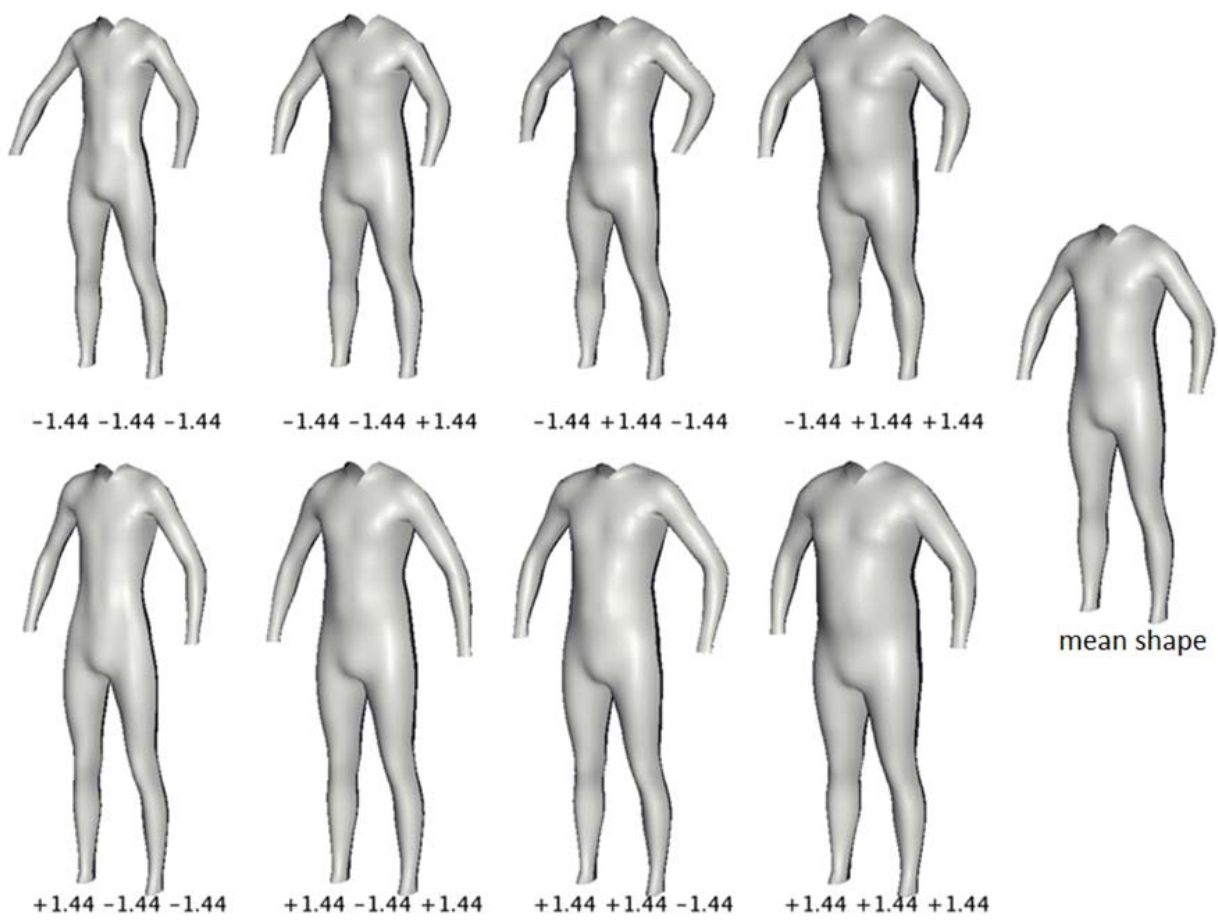

Fig. 11. Design extremes with their weights for the PC components. 


\section{Conclusion}

A database of the Netherlands Military Forces comprising of 15231 male and 2390 female scans was analyzed for inter-individual differences using principle component (PC) analysis. Based on a uniform selection along the body height and the body surface area a diverse selection of recruits was acquired. PCA models were constructed for the randomly increased subsets of 200 full body scans. After the 3D PCA fitting process of eighty PCs of the arms and body, a list of PC weights for each individual was available for further analysis. Analysis of the PCs showed that men and women differ most for stature (PC1), mass and inner leg length (PC2), and mass without inner leg length (PC3). These PC values hardly changed over the years. However, the PCs of the arms changed over the years due to changing preferences in posture.

We also used the PCA models to select digital representations of design extremes. Currently we are investigating how to design custom fit products based on such representatives of the 3D body shape.

\section{References}

1. Daanen HAM, Van De Water GJ. Whole body scanners. Displays 1998;19(3):111-120.

2. D'Apuzzo N. 3D body scanning technology for fashion and apparel industry. Proc SPIE Int Soc Opt Eng 200729 January 2007 through 30 January 2007;6491.

3. Jones PRM, Rioux M. Three-dimensional Surface Anthropometry: Applications to the Human Body. Opt Lasers Eng 1997;28(2):89-117.

4. Blackwell S, Robinette KM, Daanen HAM, Boehmer M, Fleming S, Kelly S, et al. Civilian American and European Surface Anthropometry Resource (CAESAR), Final Report, Volume II: Descriptions. AFRL-HE-WP-TR-2002-0170, United States Air Force Research Laboratory, Human Effectiveness Directorate, Crew System Interface Division, $2255 \mathrm{H}$ Street, Wright-Patterson AFB OH 45433-7022 and SAE International, 400 Commonwealth Dr , Warrendale, PA 150962002.

5. Allen B, Curless B, Popović Z. The space of human body shapes: Reconstruction and parameterization from range scans. ACM Transactions on Graphics 2003;22(3):587-594.

6. Ter Haar FB, Veltkamp RC. Expression Modeling for Expression-Invariant Face Recognition. Computers and Graphics, 2010; 34:231-241. 